\title{
Characterization of a Non-flowering Cabbage Mutant Discovered 42 Years Ago
}

\author{
Yu Kinoshita ${ }^{1}$, Ko Motoki² and Munetaka Hosokawa ${ }^{3,4 *}$ \\ ${ }^{1}$ Graduate School of Agriculture, Kyoto University, Kyoto 606-8502, Japan \\ ${ }^{2}$ Graduate School of Agriculture, Kyoto University, Kizugawa 619-0218, Japan \\ ${ }^{3}$ Faculty of Agriculture, Kindai University, Nara 631-8505, Japan \\ ${ }^{4}$ Agricultural Technology and Innovation Research Institute Kindai University (ATIRI), Nara 631-8505, Japan
}

\begin{abstract}
A non-flowering natural cabbage mutant among the open-pollinated line 'T15' was found 42 years ago. The mutant was named 'nfe' (non-flowering cabbage) and has been propagated vegetatively by cuttings. 'nfe' hardly flowers during the spring season even after plenty of low-temperature periods. This study characterized the non-flowering trait of ' $\mathrm{nfc}$ ' and assumed the mechanism. In the first experiment, we investigated the flowering characteristics of ' $\mathrm{T} 15$ ' and ' $\mathrm{nfc}$ ' over three years. Throughout the 3-year cultivation period, all ' $\mathrm{T} 15$ ' plants flowered, while the flowering ratios of ' $\mathrm{nfc}$ ' propagated by cuttings at the 1st, 2nd, and 3rd year were $0 \%, 32 \%$, and $4 \%$, respectively. In the last two years, other traits were also investigated in detail. The flowering dates of ' $\mathrm{nfc}$ ' flowering plants were later than those of ' $\mathrm{T} 15$ ', and the average numbers of flowering shoots per flowering plant of ' $\mathrm{nfc}$ ' were lower than those of ' $\mathrm{T} 15$ '. Moreover, the terminal bud of ' $\mathrm{nfc}$ ' flowering plants continued to grow vegetatively, even when their lateral shoots flowered. In the second experiment, to verify the hypothesis that 'nfe' is a chimeric plant, we investigated protoplast-regenerated plants' flowering characteristics from mesophyll protoplasts of ' $\mathrm{nfc}$ '. We obtained colonies derived from different protoplasts of ' $\mathrm{nfc}$ ' and regenerated plants from each colony (nfcPP lines). Suppose the ' $\mathrm{nfc}$ ' is a chimeric plant, in that case, protoplast-regenerated plants' flowering characteristics should be the same for each colony they derived. However, both flowering and non-flowering plants appeared in the same nfcPP lines derived from a single colony. From this, we concluded that ' $n f$ ' ' is not a chimeric plant on flowering characteristics. These results indicate that ' $\mathrm{nfc}$ ' is a mutant that maintains its flowering ability, but its flowering is strongly suppressed by some factors other than its structural characteristics.
\end{abstract}

Key Words: Brassica oleracea, chimera, flowering suppression, protoplast regeneration.

\section{Introduction}

A non-flowering natural cabbage (Brassica oleracea var. capitata) mutant among the open-pollinated line 'T15' was found in an open field 42 years ago by Prof. Susumu Yazawa. The mutant was discovered because it did not bolt and flower at all in spring, whereas the other ' $\mathrm{T} 15$ ' plants around it flowered vigorously. The mutant was named 'nfc' (non-flowering cabbage). Cabbages require a long-term low-temperature exposure for flowering, same as many other Brassicaceae

Received; January 11, 2021. Accepted; March 26, 2021.

First Published Online in J-STAGE on June 5, 2021.

This work supported by a Grant-in-Aid for JSPS Research Fellow (No. 20J23812) from the Japan Society for the Promotion of Science.

* Corresponding author (E-mail: mune@nara.kindai.ac.jp). crops (Friend, 1985). However, 'nfc' hardly flowers in the spring season even after plenty of low-temperature periods in our preliminary observation (Fig. 1; photographs provided by S. Yazawa). When 'nfc' and a commercial cabbage cultivar 'Succession', which belongs to the same succession varietal group as ' $\mathrm{T} 15$ ', were transplanted simultaneously in autumn, 'Succession' flowered vigorously in May, whereas 'nfc' continued to grow vegetatively (Fig. 1A). Furthermore, when 'nfc' was cultivated under natural conditions for approximately four years for long-term observation of 'nfc', it continued to grow vegetatively despite being exposed to low temperatures during winter and the stem length reached a height of about $1.8 \mathrm{~m}$ (Fig. 1B). Thus, 'nfc' is a mutant that hardly flowers even under flowerinductive conditions in which ordinary cabbages flower. Therefore, ' $n f c$ ' has been propagated vegetatively by 


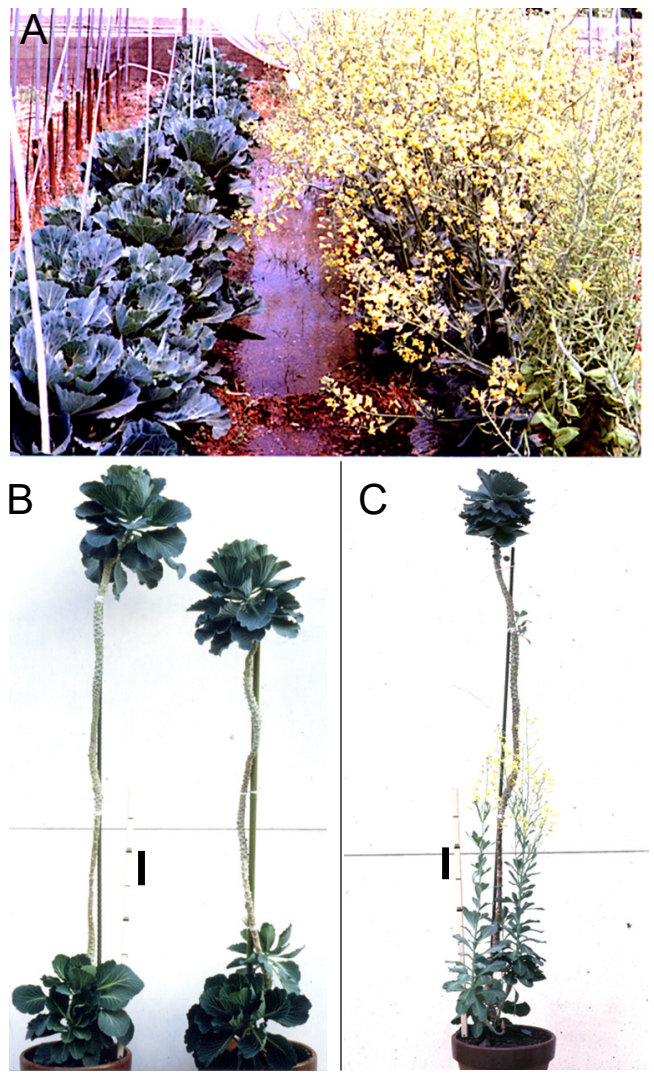

Fig. 1. (A) 'nfc' (left) and a commercial cabbage cultivar 'Succession' (right), which were transplanted simultaneously in autumn. The photograph was taken in May. 'Succession' flowered vigorously in May, whereas 'nfc' continued to grow vegetatively. (B) 'nfc' that was cultivated for about four years under natural conditions. It continued to grow vegetatively despite being exposed to low temperature during winter and the stem length reached a height of about $1.8 \mathrm{~m}$. Scale bar $=10 \mathrm{~cm}$. (C) Lateral shoots generated near the base of the main stem of ' $\mathrm{nfc}$ ' cultivated for a long-time period flowered occasionally in spring. Scale bar $=10 \mathrm{~cm}$.

cuttings in our laboratory for over 40 years.

Alternatively, a phenomenon where lateral shoots generated near the base of the main stem of 'nfc' flowered in spring has been occasionally found in our preliminary observations (Fig. 1C). We hypothesized that 'nfc' is a chimeric plant composed of non-mutated cells with flowering ability and mutated cells without flowering ability because this hypothesis could explain the occasional flowering phenomenon of ' $\mathrm{nfc}$ '. A shoot apical meristem (SAM) of angiosperms comprises three histogen layers called L1, L2, and L3 from the outer layers (Tilney-Bassett, 1963). Each of L1 and L2 is a cell layer that undergoes almost only anticlinal cell division. In contrast, L3 undergoes cell division in random directions, so the three histogen layers are of different cell lineages (Satina et al., 1940). Genetic mutation usually occurs in a cell and spreads only in the histogen layer, where the mutated cell is located. Therefore, the plant develops into a periclinal chimera consisting of mutated and non-mutated cells. Although periclinal chimeric plants can be propagated stably, spontaneous periclinal cell division and invasion to the next cell layer generate a different chimeric structure or a non-chimeric plant (Marcotrigiano, 1996). When this change occurs in SAM, bud sports with a different chimeric or non-chimeric structure arise (Marcotrigiano, 1996). Suppose ' $n f c$ ' is a periclinal chimera that contains mutated and non-mutated cells in the SAM, the spontaneous flowering phenomenon can be explained by the cell lineage changes.

This study characterized the non-flowering trait of 'nfc' and assumed the mechanism. This paper is the first report on 'nfc'. In the first experiment, to confirm the reproducibility of the flowering characteristics of ' $n f c$ ' in the preliminary observation, we cultivated dozens of ' $\mathrm{nfc}$ ' and 'T15' plants in an open field. We investigated the flowering ratios over three years. Additionally, to compare ' $\mathrm{nfc}$ ' flowering plants to ' $\mathrm{T} 15$ ', we investigated the flowering date, the number of flowering shoots, and the flower bud development ratio of the terminal bud for the last two years. In the second experiment, to verify the hypothesis that ' $\mathrm{nfc}$ ' is a chimeric plant on flowering characteristics, we evaluated the flowering characteristics of plants regenerated from each protoplast. Suppose 'nfe' is a chimeric plant composed of non-mutated and mutated cells, whether the regenerated plants flower or not will depend on the colony derived from a single protoplast with a different genetic background. Alternatively, if ' $\mathrm{nfc}$ ' is not a chimeric plant, flowering and non-flowering plants should be mixed even if they are derived from one colony.

\section{Materials and Methods}

\section{Plant materials}

'nfc' plants propagated in vitro by cuttings were used. To eliminate endophytes from 'nfc' for protoplast culture, plant regeneration of ' $\mathrm{nfc}$ ' from leaf primordium (LP)-free SAMs was conducted according to Hosokawa et al. (2004) (Fig. S1). Since 'nfc' plants propagated by cuttings and 'nfc' plants regenerated from LP-free SAMs showed similar flowering characteristics from a preliminary test, we regarded them as the same 'nfc' plants (Table S1; Fig. S1). 'T15' plants propagated in vitro by cuttings or open-pollinated ' $\mathrm{T} 15$ ' seeds were used as control (Fig. S1). We also used 'Ruby ball', 'Yumegoromo', 'Harunami' (Takii Seed Co., Ltd., Kyoto, Japan), 'Early ball', 'Kinkei No. 201' (Sakata Seed Corp., Yokohama, Japan), 'Matsunami' (Ishii Seed Growers Co., Ltd., Shizuoka, Japan), 'Watanabe-seiko No. 1', 'Yoshin', and 'Ogata Copenhagen market' (Genebank project, NARO, Japan) as commercial cultivars (Table S2).

\section{Protoplast culture and plant regeneration}

Protoplast culture was conducted according to Pelletier et al. (1983) and Hegazi and Matsubara (1992) 
with some modifications. Protoplasts were isolated from leaves of ' $n f c$ ' plants regenerated from LP-free SAMs. About $1.5 \mathrm{~g}$ of true leaves were placed on a glass petri dish in $30 \mathrm{~mL}$ cell and protoplast washing (CPW) salt solution with $91 \mathrm{~g} \cdot \mathrm{L}^{-1} \mathrm{D}(-)$-mannitol (Fujifilm Wako Pure Chemical Corp., Osaka, Japan) at pH 5.8 (Table S3). These leaves were chopped finely using a razor blade in a CPW salt solution and incubated for $30 \mathrm{~min}$ in the dark at room temperature. Then, leaves were transferred into $15 \mathrm{~mL}$ of CPW salt solution containing $10 \mathrm{~g} \cdot \mathrm{L}^{-1}$ cellulase Onozuka R-10 (Yakult Pharmaceutical Industry Co., Ltd., Tokyo, Japan) and $1 \mathrm{~g} \cdot \mathrm{L}^{-1}$ pectolyase $\mathrm{Y}-23$ (Seishin Enterprise Co., Ltd., Tokyo, Japan) and shaken at $45 \mathrm{rpm}$ for $3 \mathrm{~h}$ in the dark at $28^{\circ} \mathrm{C}$ to digest cell walls and isolate protoplasts. The CPW salt solution containing isolated protoplasts was filtered through a $50 \mu \mathrm{m}$ pore-size nylon mesh, and the filtrate was dispensed into centrifuge tubes in $2-2.5 \mathrm{~mL}$ aliquots. These tubes were centrifuged at $80 \times \mathrm{g}$ at $15^{\circ} \mathrm{C}$ for $3 \mathrm{~min}$, and the supernatant was discarded. The precipitated protoplasts were suspended in $2-2.5 \mathrm{~mL}$ of fresh CPW salt solution and centrifuged under the same conditions, and the supernatant was discarded. After this step was repeated, protoplasts were suspended in a culture medium containing 1/2 modified Murashige and Skoog (MS) medium without $\mathrm{SO}_{4}^{-}$and with $1 / 8 \mathrm{NH}_{4} \mathrm{NO}_{3}, 15 \mathrm{~g} \cdot \mathrm{L}^{-1}$ sucrose, $\quad 91 \mathrm{~g} \cdot \mathrm{L}^{-1} \quad \mathrm{D} \quad(-)$-mannitol, $2.5 \mathrm{ppm} \quad 2,4-$ dichlorophenoxyacetic acid (2,4-D), 1 ppm benzyl adenine (BA), and $0.1 \mathrm{ppm}$ 1-naphthylacetic acid (NAA) (Table S3). Equal amounts of culture medium with protoplasts and gellan gum solution containing $6 \mathrm{~g} \cdot \mathrm{L}^{-1}$ gellan gum (Fujifilm Wako Pure Chemical) and $72 \mathrm{~g} \cdot \mathrm{L}^{-1} \mathrm{D}$ $(-)$-mannitol were mixed and $2 \mathrm{~mL}$ each was dispensed into $6-\mathrm{cm}$ petri dishes. This medium in which protoplasts were suspended was semisolid. The protoplasts were cultured at $25^{\circ} \mathrm{C}$ in the dark, and when colonies that had undergone cell division to 16 cells or more were confirmed, approximately $0.5 \mathrm{~mL}$ of adding medium 1 (Table S3) was added to each petri dish. Approximately $0.5 \mathrm{~mL}$ of adding mediums 2 and 3 (Table S3) were added every other week to reduce the osmotic pressure of the medium. Calluses that grew to approximately $1 \mathrm{~mm}$ due to repeated cell divisions were placed on regeneration medium (Table S3: 1/2 MS medium without $\mathrm{SO}_{4}^{-}$and with $1 / 8 \mathrm{NH}_{4} \mathrm{NO}_{3}, 20 \mathrm{~g} \cdot \mathrm{L}^{-1}$ sucrose, $1 \mathrm{ppm}$ BA, $0.1 \mathrm{ppm}$ IAA) and cultured at $25^{\circ} \mathrm{C}$ with a $16 \mathrm{~h}$ day length and a light intensity of $80 \mu \mathrm{mol} \cdot \mathrm{m}^{-2} \cdot \mathrm{s}^{-1}$ (photosynthetic photon flux density [PPFD]). When adventitious buds were regenerated, the calluses were transferred to plant hormone-free modified MS medium. Regenerated shoots of length $2-3 \mathrm{~cm}$ were excised from the calluses and transplanted to a hormone-free modified MS medium for rooting. Regenerated shoots derived from the same colony were considered as the same nfcPP line and were subcultured. A total of 72 protoplast-regenerated plants derived from $12 \mathrm{nfcPP}$ lines were grown. Details of which callus and which regenerated shoot each protoplast-regenerated plant arose from are shown in Table S4.

\section{Growth conditions and investigation of flowering char- acteristics}

For raising seedlings, 6-cm diameter plastic pots or 128-hole cell trays were used. Nippi gardening soil No. 1 (Nihon Hiryo Co., Ltd., Tokyo, Japan) or commercial garden soil (Takii Seed) were used as a potting medium. All cultivation, fertilization, and pest management were conducted according to commercial practice. After transplanting, cultivation continued until July of the following year. After March of the following year after transplanting, cabbage heads were divided vertically with a knife so that the main stem could bolt and the lateral shoots could elongate.

In experiment 1 , in vitro ' $\mathrm{T} 15$ ' and ' $\mathrm{nfc}$ ' were transplanted into the potting medium and acclimated in a plant growth room at $22 \pm 2{ }^{\circ} \mathrm{C}$ with a $16 \mathrm{~h}$ day length and a light intensity of $80 \mu \mathrm{mol} \cdot \mathrm{m}^{-2} \cdot \mathrm{s}^{-1}$ (PPFD) in August or September in 2017-2019. 'T15' seeds were rinsed with $100 \%$ ethanol and sterilized in a $10 \%$ sodium hypochlorite solution (Fujifilm Wako Pure Chemical) for $15 \mathrm{~min}$ and sown in the potting medium in July or August in 2018-2019. Seeds of commercial cabbage cultivars were sterilized and sown as 'T15' seeds in August 2019. Only 'Yoshin' and 'Ogata Copenhagen market' were sown and planted in a 105hole cell tray filled with Nippi gardening soil No. 1 (Nihon Hiryo): peat moss PRO-MIX PGX (Premier Tech Ltd., QC, Canada) $=2: 1$ in volume ratio in August 2019. All seedlings were grown in a plastic greenhouse until transplanting. They were transplanted in an open field of the Kyoto farm, Kyoto University, or an open field of the Kizu farm, Kyoto University in September or October. For the plants transplanted in 2017, the flowering ratio was investigated. Plants with expanded petals during the cultivation period were defined as flowering plants, and the others were defined as nonflowering plants. For the plants transplanted in 2018 and 2019 , in addition to the flowering ratio, the flowering date, the number of flowering shoots, and the flower bud development ratio of the terminal bud were investigated. The flowering date was defined as the day when the petals expanded. In flowering plants, the terminal shoots and the primary lateral shoots from the main stem with flowers were counted as the number of flowering shoots (Fig. 2). In flowering plants, the flower bud development of the terminal bud was investigated by observing whether the terminal bud was a flower bud or a leaf bud visually (Fig. 2). The flowering ratio was calculated as the ratio of the number of flowering plants to the number of total plants, and the flower bud development ratio of the terminal bud was calculated as the number of plants with flower bud development of the terminal bud to the number of total flowering plants. 
Additionally, vegetative growth of 'nfc' plants was measured in the vegetative stage. In the growing season of 2018, the maximum plant width and the maximum leaf length of each ' $\mathrm{nfc}$ ' plant were measured on January 19, 2019. In the growing season of 2019, the maximum leaf length of each ' $n f c$ ' plant was measured at the Kizu farm $(n=6)$ on December 7, 2019 and at the Kyoto farm ( $\mathrm{n}=19)$ on December 18, 2019.

In experiment 2 , in vitro ' $\mathrm{nfc}$ ' protoplast-regenerated plants were transplanted into the potting medium in September 2017. In 2018, protoplast-regenerated plants maintained for a year without flowering in a plastic greenhouse or the plant growth room were cut into 6-cm diameter plastic pots to grow cutting seedlings. These seedlings were grown as in experiment 1 and transplanted in an open field of the Kyoto farm, Kyoto University in October and November 2017-2018. Throughout the 2-year cultivation period, the flowering ratio and the flower bud development ratio of the termi-

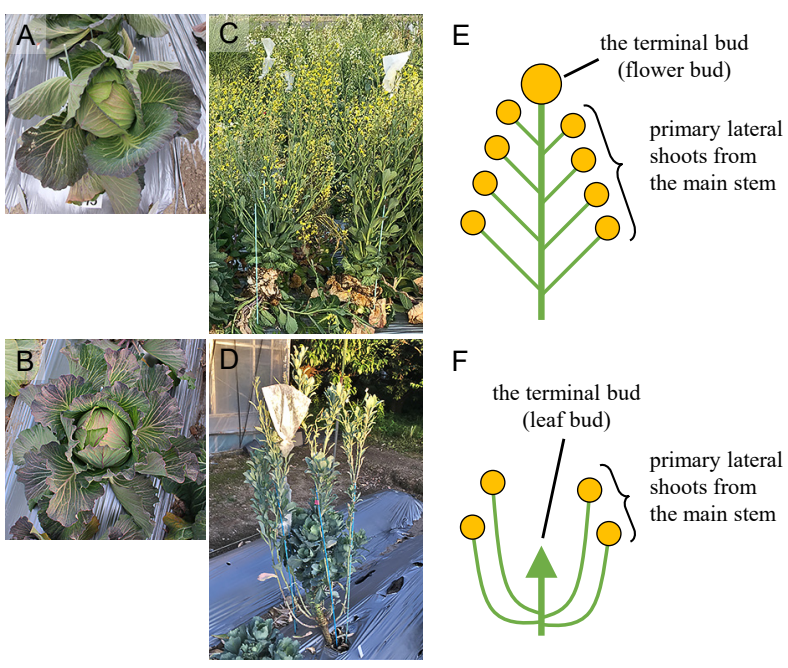

Fig. 2. A photograph of 'T15' (A) and 'nfc' (B) taken in February. A photograph of 'T15' taken in April 2020 (C) and 'nfc' propagated by cuttings taken in June 2019 (D). Schematic diagrams of plant posture of 'T15' with a flower bud (E) and 'nfc' with a leaf bud $(\mathrm{F})$ of the terminal bud. Circles indicate flower buds; the arrow indicates a leaf bud. nal bud were investigated. Additionally, the maximum plant width and the maximum leaf length of each 'nfc' protoplast-regenerated plant were measured on January 19,2019 as in experiment 1.

\section{Statistical analysis}

Statistical analyses were performed on the maximum plant widths or the maximum leaf length of the flowering and non-flowering plant of ' $\mathrm{nfc}$ ' and ' $\mathrm{nfc}$ ' protoplast-regenerated plants cultivated in 2018. Statistical analyses were performed using R v.3.6.3. (R Core Team, 2020). The Shapiro-Wilk test was used to test for normality, and the Bartlett test was used to test for equivariance. All data followed a normal distribution at a $5 \%$ level of significance, and the data of the four groups were equally distributed at a $5 \%$ significance level. One-way analysis of variance (ANOVA) followed by the Tukey-Kramer test using the multcomp package (Hothorn et al., 2008) was performed to determine differences at a $5 \%$ level of significance.

\section{Results}

Flowering characteristics of 'T15' and ' $\mathrm{Ac}$ ' (Experiment 1)

All 'T15' plants flowered yearly (Table 1). 'T15' plants propagated by cuttings and ' $\mathrm{T} 15$ ' plants grown from seeds showed similar flowering characteristics (Table S1; Fig. S1), so each data was put together in Table 1. All plants of commercial cabbage cultivars also flowered in 2019 (Table S2). Alternatively, the flowering ratios of 'nfc' were $0 \%$ (0 plants/38 plants), $32 \%$ (6 plants $/ 19$ plants), and $4 \%$ ( 1 plants $/ 25$ plants $)$ in 2017, 2018, and 2019, respectively (Table 1). The median flowering dates of ' $\mathrm{T} 15$ ' plants in 2018 and 2019 were April 19 and 8, whereas those of 'nfc' flowering plants were May 5 and April 26 (Fig. 3). The average numbers of flowering shoots per ' $\mathrm{T} 15$ ' flowering plant in 2018 and 2019 were 27.4 and 24.1, whereas those of 'nfc' flowering plants were 4.8 and 4.0, which were much lower compared with 'T15' (Fig. 4). In 'nfc' flowering plants, only the lateral shoots flowered (Figs. 1C and 2). Therefore, we confirmed the flower-

Table 1. Flowering ratios and flower bud development ratios of the terminal bud of 'T15' and 'nfc'.

\begin{tabular}{ccccccc}
\hline & \multicolumn{3}{c}{ Flowering ratio (\%) } & & \multicolumn{2}{c}{ Flower bud development ratio of the terminal bud (\%) } \\
\cline { 2 - 3 } \cline { 7 - 7 } & 2017 & 2018 & 2019 & & 2018 & 2019 \\
\hline 'T15' & $100(15 / 15)^{\mathrm{z}}$ & $100(25 / 25)$ & $100(29 / 29)$ & & $100(20 / 20)^{\mathrm{xw}}$ & $100(29 / 29)$ \\
'nfc' & $0(0 / 38)$ & $32(6 / 19)^{\mathrm{y}}$ & $4(1 / 25)$ & & $0(0 / 2)^{\mathrm{v}}$ & $0(0 / 1)$ \\
\hline
\end{tabular}

${ }^{z}$ Figures in parentheses indicate the ratio of the number of flowering plants to the number of total plants.

y Two aborted plants in which flower buds were borne but development of the flowers ceased in the middle were included in non-flowering plants.

${ }^{\mathrm{x}}$ Figures in parentheses indicate the ratio of the number of plants with flower bud development of the terminal bud to the number of total flowering plants.

w For five flowering plants of 'T15', the status of the terminal bud could not be confirmed because the terminal bud was accidentally lost due to cutting or rotting.

v For four flowering plants of 'nfc', the status of the terminal bud could not be confirmed because the terminal bud was accidentally lost due to cutting or rotting. 


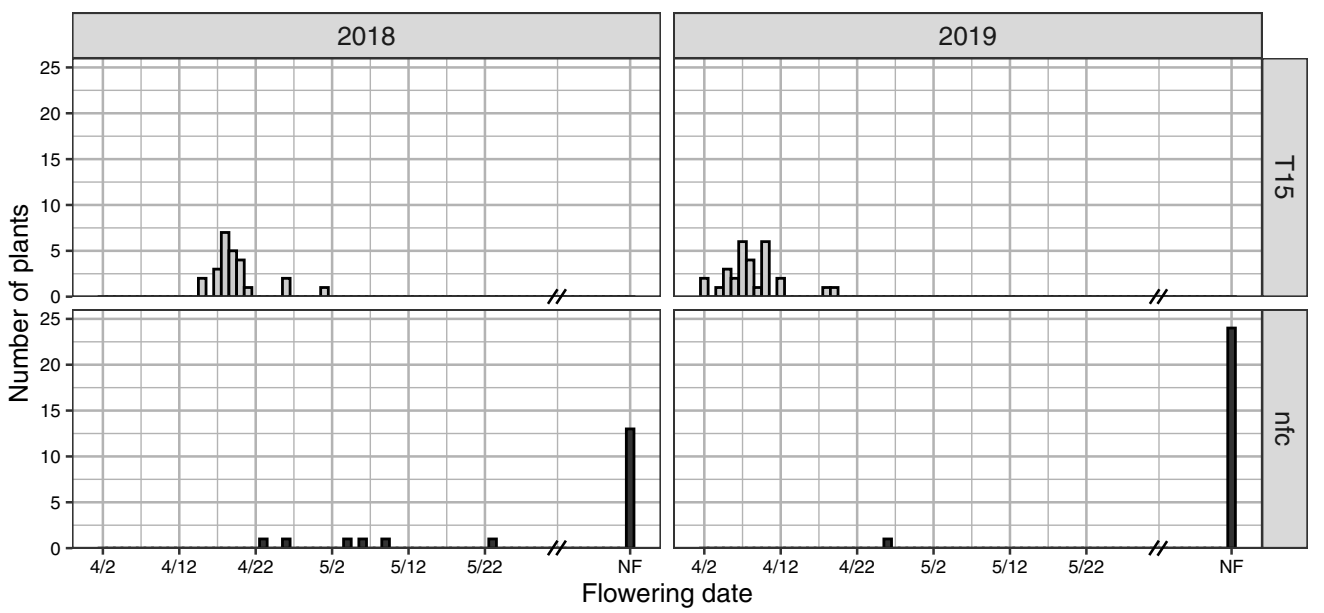

Fig. 3. The flowering dates of ' $\mathrm{T} 15$ ' and ' $\mathrm{nfc}$ ' in 2018 and 2019. "NF" represents non-flowering. The flowering date is shown as month/day. Upper panels show the number of plants of ' $\mathrm{T} 15$ ' and lower panels show that of 'nfc'.

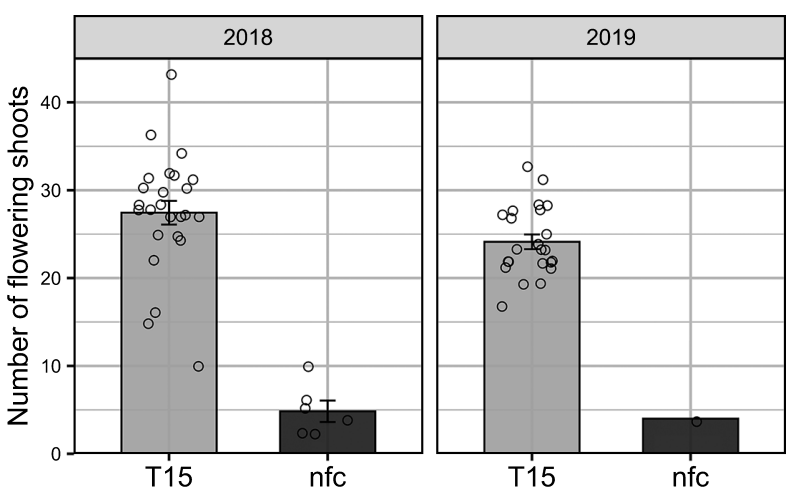

Fig. 4. The number of flowering shoots per flowering plant of 'T15' ( $n=20,2018 ; n=23,2019)$ and ' $n f c '$ ' $n=6,2018 ; n=$ 1, 2019). The flowering shoots of 'T15' cultivated in Kizu farm in $2019(n=6)$ were not counted. The values are expressed as means $\pm \mathrm{SE}$.

ing bud development of the terminal bud in flowering plants (Fig. 2). The flowering bud development ratios of the terminal bud of ' $\mathrm{T} 15$ ' were $100 \%$ in 2018 and 2019 , whereas those of 'nfc' flowering plants were both $0 \%$ (Table 1). The flowering lateral shoots of 'nfc' were generated below the node of the head leaves. The terminal bud of 'nfc' flowering plants continued to grow vegetatively until July.

Flowering characteristics of ' $n f c$ ' protoplastregenerated plants (Experiment 2)

All protoplasts precipitated during centrifugation were chloroplast-containing mesophyll cells (Fig. S2A). Some of the cultured protoplasts divided into two cells within three days to one week and then slowly continued to divide (Fig. S2B-C). Colonies grew to approximately $100 \mu \mathrm{m}$ in diameter approximately one month after protoplast isolation (Fig. S2D) and grew to white calluses of approximately $1 \mathrm{~mm}$ approximately two months after protoplast isolation. The calluses greened almost one week after being placed on the regeneration medium under a lighting condition and grew to almost $5 \mathrm{~mm}$ with adventitious buds approximately one month later (Fig. S2E). Multiple shoots were generated from the callus approximately one month after the calluses were transferred to the plant hormone-free MS medium (Fig. S2F).

For two years, $12 \mathrm{nfcPP}$ lines were investigated for flowering characteristics. There were seven nfcPP lines (nfcPP \#3, \#4, \#6, \#7, \#8, \#11, and \#17) in which flowering plants did not appear and five nfcPP lines (nfcPP $\# 1, \# 2, \# 5$, \#9, and \#10) in which flowering plants appeared (Table 2). However, non-flowering plants were present in all the nfcPP lines. The flowering ratios of the nfcPP lines in which flowering plants appeared were from low to high, and nfcPP lines \#2 and \#5 had the highest flowering ratios among them. The total flowering ratios of nfcPP lines \#2 and \#5 in 2017 and 2018 were $62 \%$ ( 8 plants/13 plants) and $75 \%$ (3 plants/4 plants), respectively. Additionally, many flowering protoplast-regenerated plants had their terminal bud with flower buds (Table 2).

\section{Discussion}

All 'T15' plants and commercial cabbage cultivars flowered (Tables 1 and S2). Therefore, the cultivation conditions in this experiment were suitable for cabbage to flower. Alternatively, the flowering ratios of ' $\mathrm{nfc}$ ' in 2017, 2018, and 2019 were $0 \%, 32 \%$, and 4\%, respectively (Table 1). Since the 'nfc' plants flowered in spring after winter like other cabbages, low-temperature exposure was considered necessary for ' $\mathrm{nfc}$ ' plants to flower. Unlike other cabbages, in 'nfc' flowering plants, only the lateral shoots generated near the base of the main stem flowered, and the terminal bud continued to grow vegetatively (Table 1; Fig. 2). Consistent with long-term observation (Fig. 1), it was very difficult for 'nfc' to flower, but lateral shoots of 'nfc' occasionally 
Table 2. Flowering ratios and flower bud development ratios of the terminal bud of nfcPP line.

\begin{tabular}{|c|c|c|c|c|}
\hline & \multicolumn{2}{|c|}{ Flowering ratio (\%) } & \multicolumn{2}{|c|}{ Flower bud development ratio of the terminal bud (\%) } \\
\hline & 2017 & 2018 & 2017 & 2018 \\
\hline nfcPP \#1 & $0(0 / 5)^{\mathrm{z}}$ & $13(1 / 8)$ & - & $-^{\mathrm{w}}$ \\
\hline nfcPP \#2 & $100(4 / 4)$ & $44(4 / 9)$ & $100(4 / 4)^{\mathrm{x}}$ & $67(2 / 3)^{\mathrm{w}}$ \\
\hline nfcPP \#3 & $0(0 / 4)$ & $0(0 / 6)$ & - & - \\
\hline nfcPP \#4 & $0(0 / 3)$ & $0(0 / 4)$ & - & - \\
\hline nfcPP \#5 & $100(1 / 1)$ & $67(2 / 3)$ & $100(1 / 1)$ & $50(1 / 2)$ \\
\hline nfcPP \#6 & $-\mathrm{y}$ & $0(0 / 3)$ & - & - \\
\hline nfcPP \#7 & - & $0(0 / 1)$ & - & - \\
\hline nfcPP \#8 & - & $0(0 / 3)$ & - & - \\
\hline nfcPP \#9 & - & $50(2 / 4)$ & - & - $^{\mathrm{w}}$ \\
\hline $\mathrm{nfcPP} \# 10$ & - & $20(1 / 5)$ & - & $0(0 / 1)$ \\
\hline nfcPP \#11 & - & $0(0 / 8)$ & - & - \\
\hline nfcPP \#17 & - & $0(0 / 1)$ & - & - \\
\hline
\end{tabular}

${ }^{\mathrm{z}}$ Figures in parentheses indicate the ratio of the number of flowering plants to the number of total plants.

y No data.

${ }^{\mathrm{x}}$ Figures in parentheses indicate the ratio of the number of plants with flower bud development of the terminal bud to the number of total flowering plants.

${ }^{\text {w }}$ For some flowering plants, the status of the terminal bud could not be confirmed because the terminal bud was accidentally lost due to cutting or rotting.

flowered. Flowering dates of ' $\mathrm{nfc}$ ' flowering plants were later than those of ' $\mathrm{T} 15$ ', and the average numbers of flowering shoots per flowering plant of 'nfc' were lower than those of 'T15' (Figs. 3 and 4). Therefore, it shows that even if 'nfc' plants flower, they do not flower in the same way as 'T15'.

The hypothesis that ' $\mathrm{nfc}$ ' is a chimeric plant composed of non-mutated cells with flowering ability and mutated cells without flowering ability could explain the occasional flowering phenomenon of ' $\mathrm{nfc}$ '. In this case, the lateral shoots generated near the base of the main stem are assumed to flower due to changes in the chimeric structure of its SAM. However, in the second experiment, both flowering and non-flowering plants appeared in 5 out of $12 \mathrm{nfcPP}$ lines (Table 2). Moreover, both flowering and non-flowering plants appeared in the protoplast-regenerated plants derived from the same regenerated shoot of the same nfcPP lines, for example, nfcPP \#1-4, nfcPP \#2-3, nfcPP \#2-4, and nfcPP \#2-5 (Table S4). Therefore, the hypothesis that ' $\mathrm{nfc}$ ' is a chimeric plant on flowering characteristics was rejected because both flowering and non-flowering plants appeared even if the genetic background was the same. From these results, it was concluded that 'nfc' is a mutant that maintains its flowering ability but its flowering is strongly suppressed.

From the above results, we assumed that 'nfc' has a genetic factor that makes it very difficult to flower, and flowering plants of ' $n f c$ ' appeared under conditions in which cabbages are easier to flower. Cabbages require long-term low-temperature exposure for flowering (Friend, 1985). Moreover, cabbage, a plantvernalization type, has a different susceptibility to low temperature depending on plant size (Ito and Saito,
1961). Therefore, we compared the temperature and plant size for each cultivation year. Monthly average temperatures in 2017-2018 were lower than those in the other two years throughout the winter season in Kyoto (Fig. S3; data were obtained from the Japan Meteorological Agency database). However, there was negligible difference in the monthly average temperature between 2018-2019 and 2019-2020, despite a big difference in the flowering ratio of ' $\mathrm{nfc}$ ' between the two seasons (Fig. S3; data were obtained from the Japan Meteorological Agency database). Furthermore, the maximum leaf length of ' $\mathrm{nfc}$ ' plants cultivated in 2019 was longer than plants cultivated in 2018, even though the investigation date was earlier in 2019 (Fig. S4A, B; not investigated in 2017). Therefore, we did not find any relationship between the annual difference in the flowering ratios of 'nfc' throughout the 3-year cultivation period and low-temperature exposure or plant size, which are conditions required for normal cabbage plants to flower. Next, we compared the plant sizes of flowering and non-flowering plants of 'nfc' cultivated in the same year. The environmental conditions for the plants cultivated in the same year can be considered to be approximately the same. Moreover, there was no significant difference in the maximum leaf length and the maximum plant width in the vegetative phase among flowering and non-flowering plants of ' $\mathrm{nfc}$ ' and those of 'nfc' protoplast-regenerated plants cultivated in 2018 (Fig. S4A, C). Accordingly, there were no differences between flowering and non-flowering plants of ' $\mathrm{nfc}$ ' cultivated in the same year, at least at this time.

Therefore, we considered the possibility that the flowering suppression in ' $\mathrm{nfc}$ ' was unstable. In other words, the hypothesis is that a change other than a 
genomic change occurs randomly at low frequency in factors involved in flowering suppression in ' $\mathrm{nfc}$ ' and this change causes 'nfc' plants to flower. Furthermore, assuming that this change is epigenetic, the occasional flowering phenomenon of ' $\mathrm{nfc}$ ' can be well explained. In micropropagated oil palm (Elaeis guineensis), approximately $5 \%$ of somatic embryo-derived palms show fruit abnormality caused by DNA hypomethylation of a long interspersed element (LINE) retrotransposon in the intron of the homeotic gene (Ong-Abdullah et al., 2015). In 'nfc' plants as well, it is possible that an epigenetic change involved in flowering suppression occurred during propagation in vitro.

The flowering ratios of nfcPP lines were significantly different for each nfcPP line, and in particular, the flowering ratios of nfcPP \#2 and \#5 were much higher than that of ' $\mathrm{nfc}$ '. When three plants (nfcPP \#2-3, \#2-4, and \#2-12) that flowered in 2017 were potted and cultivated outdoors and when three cutting seedlings and one cutting seedling derived from nfcPP \#2-4 and \#2-12, respectively, were transplanted in the open field, all plants again flowered in spring of the following year. This suggests that protoplast-regenerated plants' flowering is not due to transient stress stimulation during protoplast regeneration, but rather due to recovery of flowering ability. Flowering ratios of 'nfc' plants regenerated from LP-free SAMs, which were used in the protoplast culture, were similar to those of 'nfc' plants propagated by cuttings (Table S1). These suggest that flowering suppression in ' $\mathrm{nfc}$ ' is derepressed during protoplast regeneration. It has been reported that chromatin decondensation is induced during protoplast isolation in some plants (Zhao et al., 2001; Tessadori et al., 2007; Ondřej et al., 2009a, b). It has also been reported that reprogramming of histone $\mathrm{H} 3$ lysine 27 trimethylation (H3K27me3) is important for callus formation and that chromatin-related gene expression changes during protoplast culture in Arabidopsis thaliana (He et al., 2012; Chupeau et al., 2013). It is possible that an epigenetic change involved in flowering suppression occurred during protoplast regeneration in the nfcPP lines with a high flowering ratio. Many of the selfprogenies of flowering ' $\mathrm{nfc}$ ' protoplast-regenerated plants flowered, but some of them returned to a nonflowering state (Kinoshita et al., 2019). This reversibility of flowering/non-flowering also support that flowering of ' $\mathrm{nfc}$ ' protoplast-regenerated plants is accompanied by epigenetic changes. In the future, it will be necessary to investigate whether an epigenetic change is involved in the flowering characteristics of 'nfc' by comparing epigenomes between flowering and non-flowering plants of 'nfc' or nfcPP lines.

In conclusion, this study revealed the following about the flowering characteristics of ' $\mathrm{nfc}$ ': 1) the flowering ratio of ' $\mathrm{nfc}$ ' was very low; 2) especially, the flower bud development ratio of the terminal bud of 'nfc' was zero; 3) some flowering plants whose terminal bud flowered appeared by protoplast regeneration of ' $\mathrm{nfc}$ '; 4) the flowering protoplast-regenerated plants maintained their flowering ability in the following year; 5) 'nfc' is not a chimeric plant, and other endogenous reversible factors such as epigenetics may be involved in the instability of the flowering/non-flowering of 'nfc'.

\section{Acknowledgements}

We would like to say a special thanks to Prof. Susumu Yazawa, who discovered 'nfe' 42 years ago and provided us with precious plant material and an abundance of valuable information and photographs. Seeds of 'Watanabe-seiko No. 1' (JP No. 25974), 'Yoshin' (JP No. 37404), and 'Ogata Copenhagen market' (JP No. 25994) were kindly provided from the Genebank Project, NARO, Japan. We are grateful to Mr. Toshio Sakakibara, Mr. Katsutoshi Nonaka, Mr. Noboru Nara, and Mr. Masaru Matsuda for technical assistance with cabbage cultivation.

\section{Literature Cited}

Chupeau, M. C., F. Granier, O. Pichon, J. P. Renou, V. Gaudin and Y. Chupeau. 2013. Characterization of the early events leading to totipotency in an Arabidopsis protoplast liquid culture by temporal transcript profiling. Plant Cell 25: 2444 2463.

Friend, D. J. C. 1985. Brassica. p. 59-61. In: A. H. Halevy (ed.). Handbook of Flowering. CRC Press, Boca Raton.

He, C., X. Chen, H. Huang and L. Xu. 2012. Reprogramming of H3K27me3 is critical for acquisition of pluripotency from cultured Arabidopsis tissues. PLoS Genet. 8: e1002911. DOI: 10.1371/journal.pgen.1002911.

Hegazi, H. H. and S. Matsubara. 1992. Callus formation and plant regeneration from protoplast derived from cotyledons and hypocotyls of radish (Raphanus sativus L.) and other cruciferous plants. J. Japan. Soc. Hort. Sci. 61: 63-68.

Hosokawa, M., A. Otake, K. Ohishi, E. Ueda, T. Hayashi and S. Yazawa. 2004. Elimination of chrysanthemum stunt viroid from an infected chrysanthemum cultivar by shoot regeneration from a leaf primordium-free shoot apical meristem dome attached to a root tip. Plant Cell Rep. 22: 859-863.

Hothorn, T., F. Bretz and P. Westfall. 2008. Simultaneous inference in general parametric models. Biom. J. 50: 346-363.

Ito, H. and T. Saito. 1961. Time and temperature factors for the flower formation in cabbage. Tohoku J. Agric. Res. 12: 297 316.

Kinoshita, Y., K. Motoki, K. Shirasawa, Y. Yasui and M. Hosokawa. 2019. Flowering characteristics of progenies of hardly flowering cabbage mutant. Hort. Res. (Japan) 18 (Suppl. 2): 128 (In Japanese).

Marcotrigiano, M. 1996. Chimeras and variegation: Patterns of deceit. HortScience 32: 773-784.

Ondřej, V., M. Kitner, I. Doležalová, P. Nádvorník, B. Navrátilová and A. Lebeda. 2009a. Chromatin structural rearrangement during dedifferentiation of protoplasts of Cucumis sativus L. Mol. Cells 27: 443-447.

Ondřej, V., B. Navrátilová and A. Lebeda. 2009b. The heterochromatin as a marker for protoplast differentiation of Cucumis sativus. Plant Cell Tiss. Org. 96: 229-234.

Ong-Abdullah, M., J. M. Ordway, N. Jiang, S. E. Ooi, S. Y. Kok, N. Sarpan, N. Azimi, A. T. Hashim, Z. Ishak, S. K. Rosli, 
F. A. Malike, N. A. A. Bakar, M. Marjuni, N. Abdullah, Z. Yaakub, M. D. Amiruddin, R. Nookiah, R. Singh, E. T. L. Low, K. L. Chan, N. Azizi, S. W. Smith, B. Bacher, M. A. Budiman, A. V. Brunt, C. Wischmeyer, M. Beil, M. Hogan, N. Lakey, C. C. Lim, X. Arulandoo, C. K. Wong, C. N. Choo, W. C. Wong, Y. Y. Kwan, S. S. R. S. Alwee, R. Sambanthamurthi and R. A. Martienssen. 2015. Loss of Karma transposon methylation underlies the mantled somaclonal variant of oil palm. Nature 525: 533-537.

Pelletier, G., C. Primard, F. Vedel, P. Chetrit, R. Remy, Rousselle and M. Renard. 1983. Intergeneric cytoplasmic hybridization in cruciferae by protoplast fusion. Mol. Gen. Genet. 191: 244-250.

R Core Team. 2020. R version 3.6.3: A language and environment for statistical computing. R Foundation for Statistical Computing, Vienna, Austria. <https://www.R-project.org/>.
Satina, S., A. F. Blakeslee and A. G. Avery. 1940. Demonstrations of the three germ layers in the shoot apex of Datura by means of induced polyploidy in periclinal chimeras. Amer. J. Bot. 27: 895-905.

Tessadori, F., M. C. Chupeau, Y. Chupeau, M. Knip, S. Germann, R. V. Driel, P. Fransz and V. Gaudin. 2007. Large-scale dissociation and sequential reassembly of pericentric heterochromatin in dedifferentiated Arabidopsis cells. J. Cell Sci. 120: $1200-1208$.

Tilney-Bassett, R. A. E. 1963. The structure of periclinal chimeras. Heredity 18: 265-285.

Zhao, J., N. Morozova, L. Williams, L. Libs, Y. Avivi and G. Grafi. 2001. Two phases of chromatin decondensation during dedifferentiation of plant cells: distinction between competence for cell fate switch and a commitment for S phase. J. Biol. Chem. 276: 22772-22778. 